\title{
Time-course investigation of blood-brain barrier permeability and tight junction protein changes in a rat model of permanent focal ischemia
}

\author{
Peng Liu ${ }^{1}$ Rui Zhang ${ }^{1}$ Danyang Liu ${ }^{1}$ Jinling Wang ${ }^{1} \cdot$ Chunling Yuan ${ }^{1} \cdot$

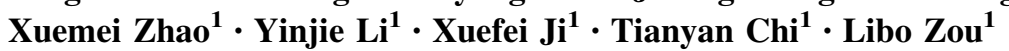

Received: 13 July 2016/Accepted: 21 December 2016/Published online: 11 January 2017

(C) The Physiological Society of Japan and Springer Japan 2017

\begin{abstract}
Permanent middle cerebral artery occlusion (pMCAO) is an animal model that is widely used to simulate human ischemic stroke. However, the timing of the changes in the expression of tight junction (TJ) proteins and synaptic proteins associated with pMCAO remain incompletely understood. Therefore, to further explore the characteristics and mechanisms of bloodbrain barrier (BBB) damage during cerebral ischemic stroke, we used a pMCAO rat model to define dynamic changes in BBB permeability within $120 \mathrm{~h}$ after
\end{abstract}

Libo Zou

807554912@qq.com

Peng Liu

liup1216@163.com

Rui Zhang

975759818@qq.com

Danyang Liu

1013780534@qq.com

Jinling Wang

1198038484@qq.com

Chunling Yuan

yutong8747@sina.com

Xuemei Zhao

84458728@qq.com

Yinjie Li

1362973376@qq.com

Xuefei Ji

jixuefei2001@163.com

Tianyan Chi

chitianyan@163.com

1 Department of Pharmacology, Shenyang Pharmaceutical University, 103 Wenhua Road, Shenhe District, Shenyang Liaoning 110016, People's Republic of China ischemia in order to examine the expression levels of the TJ proteins claudin-5 and occludin and the synaptic proteins synaptophysin (SYP) and postsynaptic density protein 95 (PSD95). In our study, Evans blue content began to increase at $4 \mathrm{~h}$ and was highest at 8 and $120 \mathrm{~h}$ after ischemia. TTC staining showed that cerebral infarction was observed at $4 \mathrm{~h}$ and that the percentage of infarct volume increased with time after ischemia. The expression levels of claudin-5 and occludin began to decline at $1 \mathrm{~h}$ and were lowest at 8 and $120 \mathrm{~h}$ after ischemia. The expression levels of SYP and PSD95 decreased from 12 to $120 \mathrm{~h}$ after ischemia. GFAP, an astrocyte marker, gradually increased in the cortex penumbra over time post-ischemia. Our study helps clarify the characteristics of pMCAO models and provides evidence supporting the translational potential of animal stroke models.

Keywords Cerebral ischemia - Blood-brain barrier - Tight junctions $\cdot$ pMCAO

\section{Introduction}

Cerebral ischemia is a major cause of death and is associated with a high incidence of acquired disability and morbidity in adults $[1,2]$. Intracranial thrombus-induced occlusion of the major cerebral artery, which decreases or completely blocks the blood flow in a specific brain region, is the pathogenesis of cerebral ischemia. Then, neurochemical and molecular events, including glutamate excitatory toxicity, $\mathrm{Ca} 2+$ overload, oxidative stress damage, the release of inflammatory cytokines, and neuronal death, lead to blood-brain barrier (BBB) leakage and brain tissue damage [3-7]. 
Structural and functional changes in brain microvascular endothelial cells and tight junctions (TJs) between the endothelial cells regulate $\mathrm{BBB}$ permeability. TJs are composed of transmembrane proteins, among which claudin-5 and occludin are the most important for BBB integrity. Decreased expression of $\mathrm{TJ}$ mRNAs and proteins directly contributes to BBB breakdown, increased vascular permeability, vasogenic edema resulting in high intracranial pressure, and high mortality risk after ischemic stroke [8-10]. Up-regulation of TJ proteins is a potential therapeutic strategy for brain stroke.

Transient and permanent middle cerebral artery occlusion (tMCAO and pMCAO) models are the animal models most commonly used to mimic human ischemic stroke. Therefore, it is important to understand the different pathophysiological changes and characteristics of these two models for evaluating novel therapeutic agents to treat human ischemic stroke. In the tMCAO model, there may be recovery from the primary core damage, and secondary delayed cell death develops after an interval of 6-12 h. However, the therapeutic time frame for human stroke is shorter than this. By contrast, in the pMCAO model, primary core damage peaks at approximately $3 \mathrm{~h}$ after MCAO [11, 12]. Jiao et al. previously investigated changes in BBB integrity, endothelial morphology, and TJ proteins over time following tMCAO in SD rats [9]. However, the timing of the changes in TJ proteins and synaptic proteins associated with pMCAO remain unclear. In this study, we use the permanent model of ischemic stroke, which eliminates the influence of recanalization to investigate changes in BBB integrity, neuronal damage, astrocyte activity, and TJ proteins over time following ischemic stroke.

\section{Materials and methods}

\section{Animal model subjects}

Adult male SD rats (200-250 g) were procured from Liao Ning Chang Sheng Biotechnology Co., Ltd. The animals were maintained under standard housing conditions (room temperature $25 \pm 1{ }^{\circ} \mathrm{C}$ and humidity $50 \pm 2 \%$ ) with a 12-h light and 12-h dark cycle. Water and food were available ad libitum. All animal studies were performed in strict accordance with legislation in P.R. China on the use and care of laboratory animals and with guidelines established by the Institute for Experimental Animals at Shenyang Pharmaceutical University (Permit Number: SYPUIACUC-C2015-0831-203). The rats were randomly divided into eight groups: sham-operated group and groups receiving pMCAO for $1,4,8,12,24,72$, or $120 \mathrm{~h}$.

\section{pMCAO}

The rats were anesthetized with chloral hydrate $(300 \mathrm{mg} /$ $\mathrm{kg}$ i.p.). Then, the right common (CCA), external (ECA), and internal carotid artery (ICA) were exposed and isolated. A monofilament nylon suture with a blunted tip $(0.26-0.28 \mathrm{~mm})$ was inserted into the right ECA and pushed into the ICA until light resistance was felt. The filament was inserted approximately 19-22 $\mathrm{mm}$ in order to block the middle cerebral artery (MCA). The filament remained inserted for $1,4,8,12,24,72$, or $120 \mathrm{~h}$, after which the animals were sacrificed. Sham group rats received the same surgery without insertion of the monofilament nylon suture.

\section{Assessment of infarct size}

Brain infarct size was evaluated using 2,3,5-triphenyltetrazolium chloride (TTC) at 1, 4, 8, 12, 24, 72, and $120 \mathrm{~h}$ after pMCAO. The rats were sacrificed, and the brains were quickly isolated. Brains were sectioned into five coronal slices (3-mm intervals), stained with a $2 \%$ solution of TTC at $37^{\circ} \mathrm{C}$ for $15 \mathrm{~min}$, and then fixed with $4 \%$ paraformaldehyde. The infarct area appeared white, and the normal area was stained red. TTC-stained sections were photographed using a digital camera, and images were analyzed using Image-Pro Plus 5.1 software. The infarct area is expressed as the ratio of the volume of the white area over that of the total brain area. The total infarct size is expressed as the percentage of damaged area of five slices over the total brain area.

\section{Measurement of $\mathrm{BBB}$ permeability}

One hour before decapitation of the rats, 2\% Evans blue $(\mathrm{EB}, 4 \mathrm{mg} / \mathrm{kg}$ ) was injected into the tail vein. The rats were anesthetized with chloral hydrate (300 mg/kg i.p.), and then normal saline was injected into the left cardiac ventricle until colorless perfusion fluid was obtained from the right atrium. The right hemisphere of the brain was exposed and isolated. Then, each hemisphere was weighed, homogenized in $2 \mathrm{ml} \mathrm{5 \%}$ trichloroacetic acid, and centrifuged $(10,000 \mathrm{r} / \mathrm{m}, 20 \mathrm{~min})$. The content of $\mathrm{EB}$ was determined via spectrophotometry (at $620 \mathrm{~nm}$ ). The results are expressed as ng/g tissue, as calculated using a standard curve.

\section{Western-blot analysis}

The rats were decapitated under excessive ether anesthesia. Ischemic penumbra tissues were isolated and stored at $-80{ }^{\circ} \mathrm{C}$. Brain issues were homogenized in $400 \mu \mathrm{l}$ of cold extraction buffer. Protein concentration of the lysate was 
determined using a BCA Protein Assay Kit (Kangwei Biotechnology, China). Equal amounts of proteins $(45 \mu \mathrm{g})$ were separated by $8-10 \%$ sodium dodecyl sulfate polyacrylamide gels, electrophoretically transferred to PVDF membranes (Millipore, USA). Membranes were blocked with $5 \%$ skim milk for $2 \mathrm{~h}$ at room temperature and then incubated overnight at $4{ }^{\circ} \mathrm{C}$ with anti-Occludin antibody (1:500, Santa Cruz, USA), anti-Claudin-5 antibody (1:500, Santa Cruz, USA), anti-SYP antibody (1:1000, Santa Cruz, USA), anti-PSD95 antibody (1:1000, Santa Cruz, USA), anti-GFAP antibody (1:500, Boster, China), or anti- $\beta$-actin antibody (1:1000, Santa Cruz, USA). After the protein was stained for $2 \mathrm{~h}$ at room temperature with HRP-conjugated secondary antibodies against mouse $\operatorname{IgG}$ and rabbit $\operatorname{IgG}$ (1:5000, Santa Cruz, USA), an ECL kit (Kangwei Biotechnology, China) was used to detected protein bands. The intensities of the bands were quantified by densitometry using Quantity One 4.6.2 software (Bio-Rad, USA) and corrected using the corresponding $\beta$-actin levels. The results are expressed as percentages of the control.

\section{Statistical analysis}

All data are presented as the mean \pm SEM. Statistical significance was determined with one-way ANOVA followed by Fisher's least significant difference (LSD) multiple comparisons test; $p<0.05$ was considered to indicate statistical significance. The data were analyzed using SPSS 17.0.

\section{Results}

\section{Changes in infarct volume after ischemia}

Brain infarct size was evaluated by TTC staining at $1,4,8,12$, 24,72 , and $120 \mathrm{~h}$ after ischemia. The infarct area appeared white, whereas the normal brain area was stained in red. Compared with the sham group, the infarct size percentage was significantly increased by $22.48 \pm 3.52,25.60 \pm 2.57$, $29.90 \pm 1.33,35.11 \pm 2.66$, and $28.50 \pm 1.86 \%$ in the 8,12 , $24,72$, and 120 h groups, respectively ( $p<0.05$, Fig. 1$)$. No significant infarct area was observed in the Sham group or in the $1 \mathrm{~h}$ post-pMCAO group. These results indicated a time-dependent increase in brain infarct injury after focal ischemia and that the infarct regions developed in both the striatum and lateral cortex.

\section{Changes in BBB permeability after ischemia}

The amount of EB leakage was measured in order to evaluate BBB permeability after ischemia in the pMCAO model. Increased EB content was observed in the ischemic regions, with the content significantly greater in the $4,8,12,24$, and

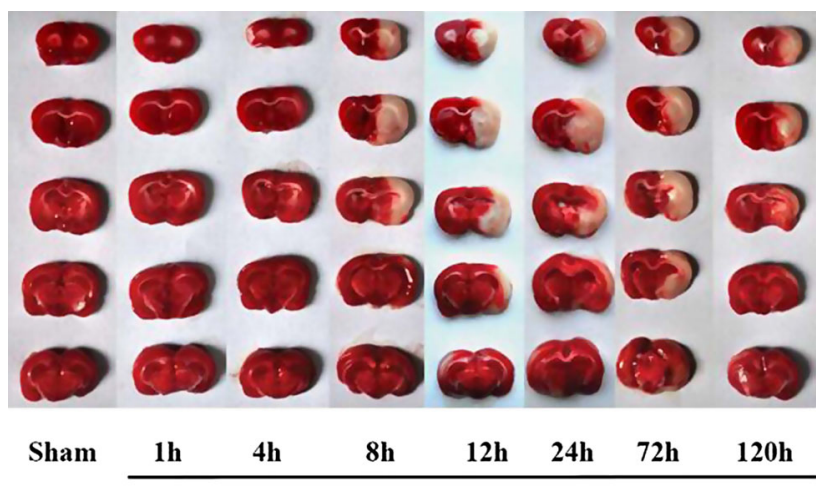

MCAO

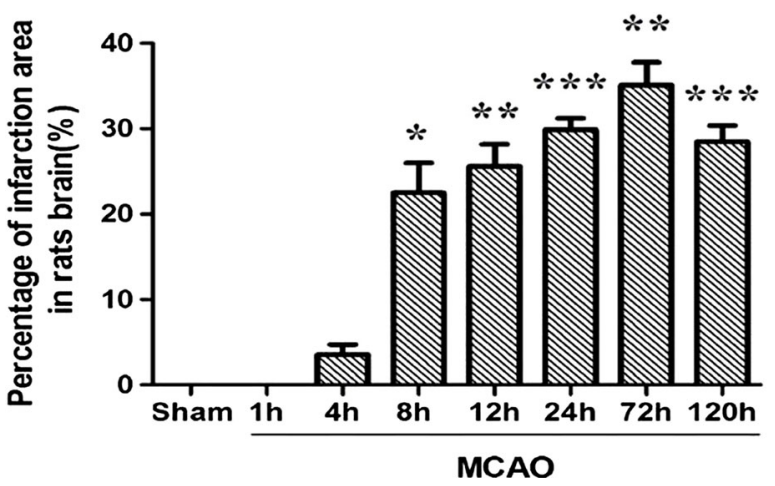

Fig. 1 Percentage of infarction area in rats brain at different time points after MCAO. Compared with the sham group, the infarct size percentage of the $8,12,24,72$, and 120 h groups were significantly increased. Data represent mean $\pm \mathrm{SEM}, \quad n=6 . * p<0.05$, ** $p<0.01, * * * p<0.001$ vs. sham group

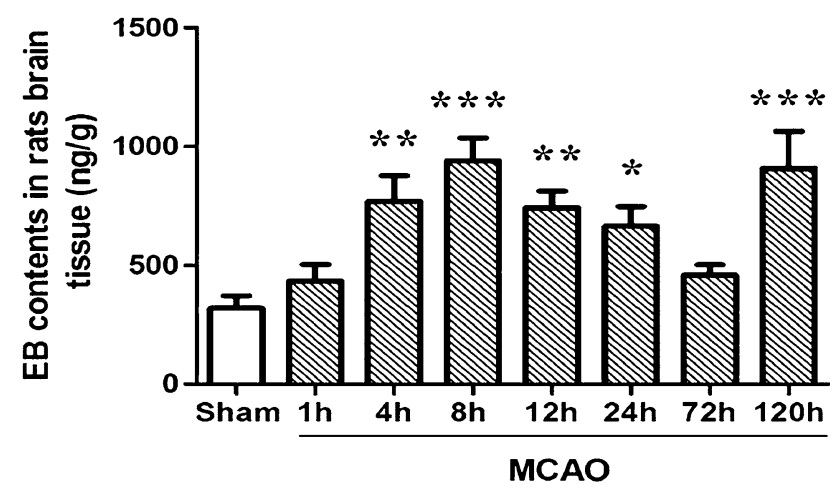

Fig. 2 Evans blue (EB) contents in brain tissue at different time points after MCAO. EB content of the 4, 8, 12, 24, and $120 \mathrm{~h}$ groups was significantly increased compared to that of the sham group. Data represent mean \pm SEM, $\quad n=6 . \quad * p<0.05, \quad * * p<0.01$, $* * * p<0.001$ vs. sham group

$120 \mathrm{~h}$ groups than in the sham group ( $p<0.05$, Fig. 2). EB content was highest at 8 and $120 \mathrm{~h}$ after ischemia.

\section{Protein expression of SYP and PSD95 after ischemia}

To assess pMCAO-induced neuronal damage, we measured the expression levels of the synaptic proteins SYP and 
PSD95 in ischemic penumbra tissues. Expression levels of SYP at $8,12,24,72$, and $120 \mathrm{~h}$ after ischemia and of PSD95 at $12,24,72$, and $120 \mathrm{~h}$ after were significantly lower than in the sham group $(p<0.01$, Fig. 3a, b).

\section{Protein expression of claudin-5 and occludin after ischemia}

To assess pMCAO-induced BBB breakdown, we measured the expression levels of the TJ proteins claudin-5 and occludin in ischemic penumbra tissues. Expression levels
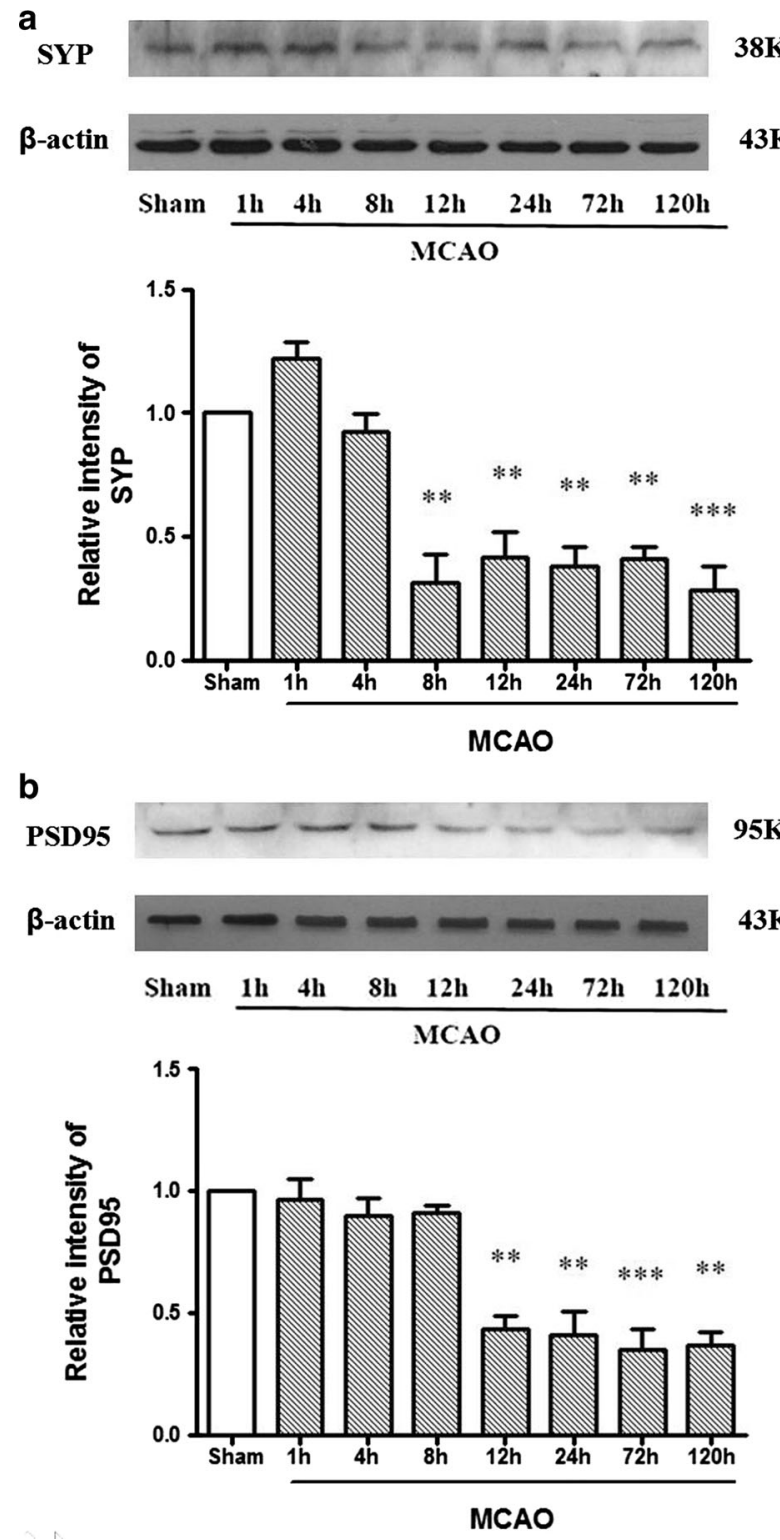

Fig. 3 The expression of SYP and PSD95 in penumbra at different time points after MCAO. Expression levels of SYP at 8, 12, 24, 72, and $120 \mathrm{~h}$ after ischemia and of PSD95 at 12, 24, 72, and $120 \mathrm{~h}$ after were significantly reduced compared to the sham group. Data represent mean \pm SEM, $n=3$. ** $p<0.01$, *** $p<0.001$ vs. sham group of claudin-5 at 8,12 , and $120 \mathrm{~h}$ after ischemia and of occludin at $8,12,24$, and $120 \mathrm{~h}$ after were significantly lower than in the sham group ( $p<0.05$, Fig. $4 \mathrm{a}, \mathrm{b})$. These data show that the integrity of the BBB was lost in ischemic rats.

\section{Protein expression of GFAP after ischemia}

To examine the activity of astrocytes, we used Western blotting to measure the expression of GFAP. Expression levels of GFAP at 12, 24, and $120 \mathrm{~h}$ after ischemia were significantly greater than in the sham group $(p<0.05$, Fig. 5).

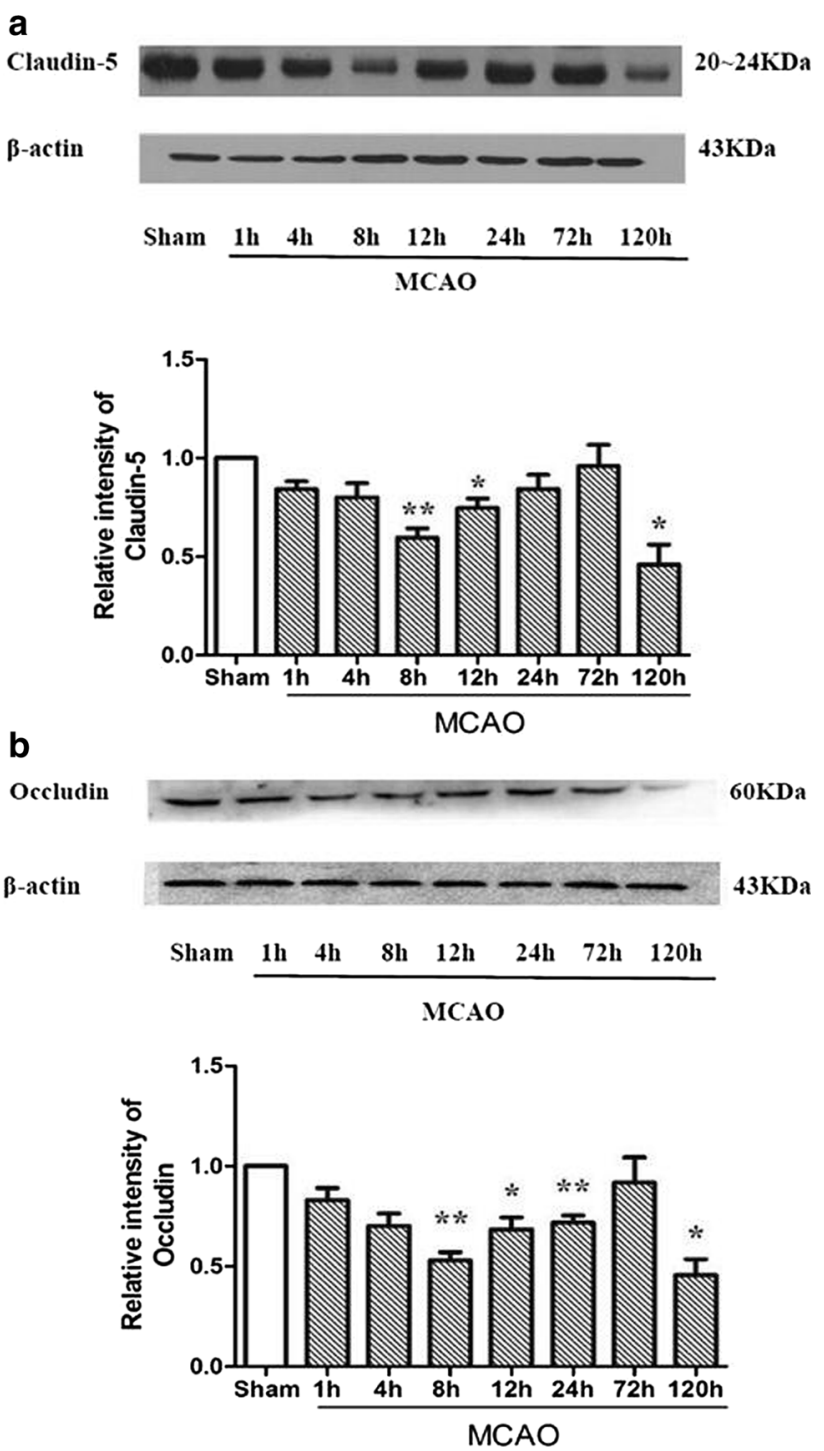

Fig. 4 The expression of Claudin-5 and Occludin in penumbra at different time points after MCAO. Expression levels of claudin-5 at 8, 12 and $120 \mathrm{~h}$ after ischemia and of occludin at 8,12, 24, and $120 \mathrm{~h}$ after were significantly reduced compared to the sham group. Data represent mean \pm SEM, $n=6$. $* p<0.05, * * p<0.01$ vs. sham group 


\section{Discussion}

In this study, we demonstrated that BBB permeability in the ischemic hemisphere of the brain gradually increased within 120-h post-ischemia. Previous studies have reported that astrocytes play key roles in maintaining the integrity and promoting the maturation of BBB [13, 14]. TJs between endothelial cells play a key role in determining vascular permeability $[15,16]$. Therefore, we characterized the time-course and relationship of the expression of the TJ proteins claudin-5 and occludin, the synaptic proteins SYP and PSD95, and the activity of astrocytes within $120 \mathrm{~h}$ during pMCAO-induced $\mathrm{BBB}$ leakage.

The BBB is the gatekeeper of the CNS, where it acts as the immunological, transport, metabolic, and physical boundary that separates the brain from systemic circulation [16-19]. Disruption of the BBB is one of the first events after ischemic stroke. After ischemia, the integrity of the BBB is destroyed, leading to the infiltration of inflammatory cells and high-molecular-weight proteins into the brain, resulting in cerebral infarction and edema [20]. Peripheral injection of Evans blue (961 Da) is the classic method for measuring BBB leakage. Evans blue binds to serum albumin, thereby becoming a tracer for highmolecular-weight molecules that can penetrate the brain after the BBB is damaged [21].

Here, we show that the integrity of the BBB in the ischemia-affected hemisphere was significantly impaired from 4 to $120 \mathrm{~h}$ after ischemia. BBB permeability was highest at 8 and $120 \mathrm{~h}$ after ischemia. This result is interesting because it is somewhat similar to a study using an
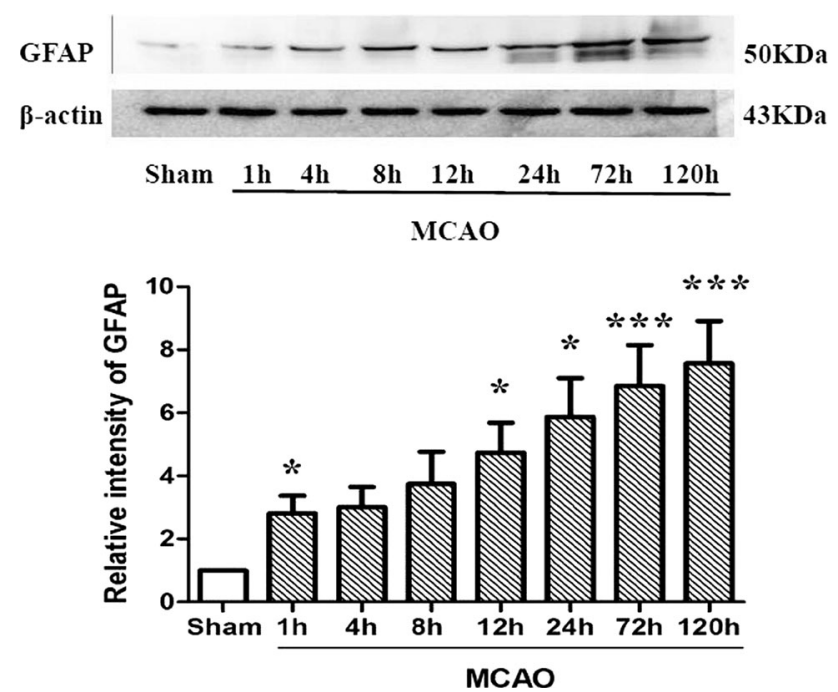

Fig. 5 The expression of GFAP in penumbra at different time points after MCAO. Expression level of GFAP at 12, 24, 72, and $120 \mathrm{~h}$ after ischemia were significantly increased compared to the sham group. Data represent mean \pm SEM, $n=6$. $* p<0.05$, *** $p<0.001$ vs. sham group ischemia-reperfusion model [9]. It is possible that the first peak of BBB permeability is during an acute hyperemia period resulting from a quick elevation in cerebral blood flow $[22,23]$. We speculate that the second peak of BBB permeability is due to the appearance of inflammatory cytokines released by microglia and astrocytes; these cytokines then damage cerebral microvessel endothelial cells [24-26]. Compared to the ischemia-reperfusion model, the permanent ischemia model causes more severe injury. The peak of Evans blue content in brain tissue is $0.94 \mu \mathrm{g} / \mathrm{g}$ in our study, whereas in the ischemia-reperfusion model the peak content is just $0.4 \mu \mathrm{g} / \mathrm{g}$ [9].

TJs are one form of intercellular adhesion structures and are responsible for sealing the intervening space in epithelial and endothelial cell sheets [27]. The TJ proteins claudin-5 and occludin perform a vital role as structural and functional components of TJs. Claudin-5, a transmembrane $\mathrm{TJ}$ protein, has been identified in BBB endothelial cells and directly determines BBB integrity $[16,28]$. Regulation of claudin-5 activity by PKA and Rho kinases has been characterized in the process of TJ permeability [29]. Increased expression of claudin-5 increases the small-molecule paracellular permeability at the BBB. Occludin is highly expressed in brain microvascular endothelial cells, and it is detected consistently along cell margins. Although several experiments have shown that occludin is not correlated with the formation of TJs, occludin is still a critical regulatory protein of BBB function [13]. The presence of occludin in the cell membrane mediates $\mathrm{TJ}$ responses to inflammation and acute $\mathrm{rCBF}$ changes [30]. Regulatory actions of occludin are influenced by PKC, connexin-26, and PI3K (p85) [31]. Based on the key roles of claudin-5 and occludin in pMCAO-induced BBB leakage, we used western blotting to assess the changes over time in the presence of these two TJ proteins in ischemic penumbra tissues of the brain within $120 \mathrm{~h}$ after ischemia. Here, we demonstrate that the expression of these proteins is significantly decreased at 8,12 , and $120 \mathrm{~h}$ after pMCAO, which is consistent with the two peaks of Evans blue leakage. The data suggest that the reduction of claudin-5 and occludin in ischemic hemisphere contributes to impairment of BBB integrity over time following focal ischemia. In this study, TJ proteins are up-regulated at $72 \mathrm{~h}$ and EB leakage is suppressed at this same time point. In cerebral ischemia models, the time point $72 \mathrm{~h}$ belongs to the subacute phase. Compared with 24 and $48 \mathrm{~h}$ after pMCAO, the motor function of rats shows slight recovery at $72 \mathrm{~h}$ in rotarod test [32]. The levels of transcription factor nuclear factor erythroid 2-related factor 2 (Nrf2) and of heme oxygenase-1 (HO-1) peak at $24 \mathrm{~h}$ and decrease at $72 \mathrm{~h}$ after ischemia, and NF- $\mathrm{KB}$ is also down-regulated at $72 \mathrm{~h}[33,34]$. In addition, the serum level of the proinflammatory factor TNF- $\alpha$ is decreased, while that of the 
anti-inflammatory cytokine IL-10 is increased at $72 \mathrm{~h}$ after ischemia [34]. Therefore, we speculate that transcription factors regulate the levels of aquaporins, TJ proteins and inflammatory cytokines, further inducing a recovery of BBB integrity. However, unlike the results for EB leakage and TJ proteins, the percentage of the infarct volume increases with time after ischemia, and the expression levels of synaptic proteins decrease. Because the infarct core expands quickly, therapeutic interventions are not completely effective if blood flow is not fully restored within the revival time after ischemia. Endothelial cells, along with other supporting cells such as neurons, pericytes, astrocytes, and microglia, determine the integrity of the BBB and are collectively known as the neurovascular unit [35]. The synthesis of GFAP by mature astrocytes can be stimulated by a large number of physical and chemical insults, and GFAP up-regulation is one of the most important features of astrogliosis [36, 37]. Our data showed that the expression of GFAP was markedly increased in a time-dependent manner, suggesting that astrocyte activation contributes to the impairment of BBB integrity after focal ischemia.

In conclusion, our data provide a time-course of $\mathrm{BBB}$ leakage and TJ protein changes within $120 \mathrm{~h}$ after permanent focal ischemia in a rat model. These results are helpful for understanding the characteristics of pMCAO and support the translational potential of animal stroke models.

Acknowledgements This work was supported by the Career Development Program for Young Teachers in Shenyang Pharmaceutical University (No. ZQN2015028), the National Natural Science Foundation of China (81503057) and the Key Laboratory for Neurodegenerative Diseases of Ministry of Education (Capital Medical University) (1300-1150170609).

\section{Compliance with ethical standards}

Ethical approval All animal procedures performed in this study were approved by the Institute for Experimental Animals at Shenyang Pharmaceutical University (Permit Number: SYPU-IACUC-C20150831-203).

Conflict of interest The authors declare that they have no conflicts of interest.

\section{References}

1. Hankey GJ (2012) Anticoagulant therapy for patients with ischaemic stroke. Nat Rev Neurol 8:319-328

2. Khaksari M, Mehrjerdi FZ, Rezvani ME, Safari F, Mirgalili A, Niknazar S (2017) The role of erythropoietin in remote renal preconditioning on hippocampus ischemia/reperfusion injury. J Physiol Sci 67:163-171

3. Sadana P, Coughlin L, Burke J, Woods R, Mdzinarishvili A (2015) Anti-edema action of thyroid hormone in MCAO model of ischemic brain stroke: possible association with AQP4 modulation. J Neurol Sci 354:37-45
4. Sahota P, Savitz SI (2011) Investigational therapies for ischemic stroke: neuroprotection and neurorecovery. Neurotherapeutics $8: 434-451$

5. Thompson JW, Narayanan SV, Koronowski KB, Morris-Blanco K, Dave KR, Perez-Pinzon MA (2015) Signaling pathways leading to ischemic mitochondrial neuroprotection. J Bioenerg Biomembr 47:101-110

6. Hazekawa M, Sakai Y, Yoshida M, Haraguchi T, Uchida T (2012) Single injection of ONO-1301-loaded PLGA microspheres directly after ischaemia reduces ischaemic damage in rats subjected to middle cerebral artery occlusion. J Pharm Pharmacol 64:353-359

7. Aboutaleb N, Shamsaei N, Khaksari M, Erfani S, Rajabi H, Nikbakht F (2015) Pre-ischemic exercise reduces apoptosis in hippocampal CA3 cells after cerebral ischemia by modulation of the $\mathrm{Bax} / \mathrm{Bcl}-2$ proteins ratio and prevention of caspase- 3 activation. J Physiol Sci 65:435-443

8. Liu X, Wang Z, Wang P, Yu B, Liu Y, Xue Y (2013) Green tea polyphenols alleviate early BBB damage during experimental focal cerebral ischemia through regulating tight junctions and PKCalpha signaling. BMC Complement Altern Med 13:187

9. Jiao H, Wang Z, Liu Y, Wang P, Xue Y (2011) Specific role of tight junction proteins claudin-5, occludin, and ZO-1 of the blood-brain barrier in a focal cerebral ischemic insult. J Mol Neurosci 44:130-139

10. Shin JA, Kim YA, Jeong SI, Lee KE, Kim HS, Park EM (2015) Extracellular signal-regulated kinase1/2-dependent changes in tight junctions after ischemic preconditioning contributes to tolerance induction after ischemic stroke. Brain Struct Funct 220:13-26

11. Fluri F, Schuhmann MK, Kleinschnitz C (2015) Animal models of ischemic stroke and their application in clinical research. Drug Des Dev Ther 9:3445-3454

12. Hossmann K-A (2012) The two pathophysiologies of focal brain ischemia: implications for translational stroke research. J Cereb Blood Flow Metab 32:1310-1316

13. Sandoval KE, Witt KA (2008) Blood-brain barrier tight junction permeability and ischemic stroke. Neurobiol Dis 32:200-219

14. Xu L, Dan M, Shao A, Cheng X, Zhang C, Yokel RA, Takemura T, Hanagata N, Niwa M, Watanabe D (2015) Silver nanoparticles induce tight junction disruption and astrocyte neurotoxicity in a rat blood-brain barrier primary triple coculture model. Int $\mathbf{J}$ Nanomed 10:6105-6118

15. Cen J, Liu L, Li MS, He L, Wang LJ, Liu YQ, Liu M, Ji BS (2013) Alteration in P-glycoprotein at the blood-brain barrier in the early period of MCAO in rats. J Pharm Pharmacol 65:665-672

16. Zhang L, Zhao H, Zhang X, Chen L, Zhao X, Bai X, Zhang J (2013) Nobiletin protects against cerebral ischemia via activating the p-Akt, p-CREB, BDNF and Bcl-2 pathway and ameliorating BBB permeability in rat. Brain Res Bull 96:45-53

17. Persidsky Y, Ramirez SH, Haorah J, Kanmogne GD (2006) Blood-brain barrier: structural components and function under physiologic and pathologic conditions. J Neuroimmune Pharmacol 1:223-236

18. Cui L, Zhang X, Yang R, Wang L, Liu L, Li M, Du W (2010) Neuroprotection of early and short-time applying atorvastatin in the acute phase of cerebral ischemia: down-regulated 12/15LOX, p38MAPK and cPLA2 expression, ameliorated BBB permeability. Brain Res 1325:164-173

19. Singh DP, Nimker C, Paliwal P, Bansal A (2016) Ethyl 3, 4-dihydroxybenzoate (EDHB): a prolyl hydroxylase inhibitor attenuates acute hypobaric hypoxia mediated vascular leakage in brain. J Physiol Sci 66:315-326

20. Liu R, Yuan H, Yuan F, Yang SH (2012) Neuroprotection targeting ischemic penumbra and beyond for the treatment of ischemic stroke. Neurol Res 34:331-337 
21. Bonova P, Danielisova V, Nemethova M, Matiasova M, Bona M, Gottlieb M (2015) Scheme of ischaemia-triggered agents during brain infarct evolution in a rat model of permanent focal ischaemia. J Mol Neurosci 57:73-82

22. Huang ZG, Xue D, Preston E, Karbalai H, Buchan AM (1999) Biphasic opening of the blood-brain barrier following transient focal ischemia: effects of hypothermia. Can J Neurol Sci 26:298-304

23. Kuroiwa T, Ting P, Martinez H, Klatzo I (1985) The biphasic opening of the blood-brain barrier to proteins following temporary middle cerebral artery occlusion. Acta Neuropathol 68:122-129

24. Kim ST, Doo AR, Kim SN, Kim SY, Kim YY, Kim JH, Lee H, Yin CS, Park HJ (2012) Acupuncture suppresses kainic acidinduced neuronal death and inflammatory events in mouse hippocampus. J Physiol Sci 62:377-383

25. O'Carroll SJ, Kho DT, Wiltshire R, Nelson V, Rotimi O, Johnson R, Angel CE, Graham ES (2015) Pro-inflammatory TNF $\alpha$ and IL$1 \beta$ differentially regulate the inflammatory phenotype of brain microvascular endothelial cells. J Neuroinflammation 12:131

26. Matsumoto J, Dohgu S, Takata F, Nishioku T, Sumi N, Machida T, Takahashi H, Yamauchi A, Kataoka Y (2012) Lipopolysaccharide-activated microglia lower P-glycoprotein function in brain microvascular endothelial cells. Neurosci Lett 524:45-48

27. Nicolazzo JA, Charman SA, Charman WN (2006) Methods to assess drug permeability across the blood-brain barrier. J Pharm Pharmacol 58:281-293

28. Chen L, Wang L, Zhang X, Cui L, Xing Y, Dong L, Liu Z, Li Y, Zhang X, Wang C, Bai X, Zhang J, Zhang L, Zhao X (2012) The protection by Octreotide against experimental ischemic stroke: up-regulated transcription factor Nrf2, HO-1 and down-regulated NF- $\kappa \mathrm{B}$ expression. Brain Res 1475:80-87

29. Yamamoto M, Ramirez SH, Sato S, Kiyota T, Cerny RL, Kaibuchi K, Persidsky Y, Ikezu T (2008) Phosphorylation of claudin-
5 and occludin by rho kinase in brain endothelial cells. Am J Pathol 172:521-533

30. Balda MS, Whitney JA, Flores C, González S, Cereijido M, Matter K (1996) Functional dissociation of paracellular permeability and transepithelial electrical resistance and disruption of the apical-basolateral intramembrane diffusion barrier by expression of a mutant tight junction membrane protein. J Cell Biol 134:1031-1049

31. Nusrat A, Parkos CA, Verkade P, Foley CS, Liang TW, InnisWhitehouse W, Eastburn KK, Madara JL (2000) Tight junctions are membrane microdomains. J Cell Sci 113:1771-1781

32. Sayeed I, Wali B, Stein DG (2007) Progesterone inhibits ischemic brain injury in a rat model of permanent middle cerebral artery occlusion. Restor Neurol Neurosci 25:151-159

33. Yang C, Zhang X, Fan H, Liu Y (2009) Curcumin upregulates transcription factor Nrf2, HO-1 expression and protects rat brains against focal ischemia. Brain Res 1282:133-141

34. Zuo L, Shi L, Yan F (2016) The reciprocal interaction of sympathetic nervous system and cAMP-PKA-NF-kB pathway in immune suppression after experimental stroke. Neurosci Lett 627:205-210

35. Noda M, Kobayashi AI (2016) Nicotine inhibits activation of microglial proton currents via interactions with $\alpha 7$ acetylcholine receptors. J Physiol Sci. doi:10.1007/s12576-016-0460-5

36. Chen XH, Lin ZZ, Liu AM, Ye JT, Luo Y, Luo YY, Mao XX, Liu PQ, Pi RB (2010) The orally combined neuroprotective effects of sodium ferulate and borneol against transient global ischaemia in C57 BL/6J mice. J Pharm Pharmacol 62:915-923

37. Yamaguchi M, Seki T, Imayoshi I, Tamamaki N, Hayashi Y, Tatebayashi Y, Hitoshi S (2016) Neural stem cells and neuro/ gliogenesis in the central nervous system: understanding the structural and functional plasticity of the developing, mature, and diseased brain. J Physiol Sci 66:197-206 